\title{
Edge mode coupling within a plasmonic
}

\section{nanoparticle - Supporting Information}

Franz-Philipp Schmidt, ${ }^{*},+\ddagger$ Harald Ditlbacher, ${ }^{\dagger}$ Andreas Hohenau ${ }^{\dagger}$ Ulrich

Hohenester, ${ }^{\dagger}$ Ferdinand Hofer, ${ }^{\ddagger}$ and Joachim R. Krenn ${ }^{\dagger}$

Institute of Physics, University of Graz, Austria, and Institute for Electron Microscopy and

Nanoanalysis, Graz University of Technology, Austria

E-mail: franz.schmidt@uni-graz.at

${ }^{*}$ To whom correspondence should be addressed

${ }^{\dagger}$ Institute of Physics, University of Graz, Austria

${ }_{\ddagger}^{\ddagger}$ Institute for Electron Microscopy and Nanoanalysis, Graz University of Technology, Austria 

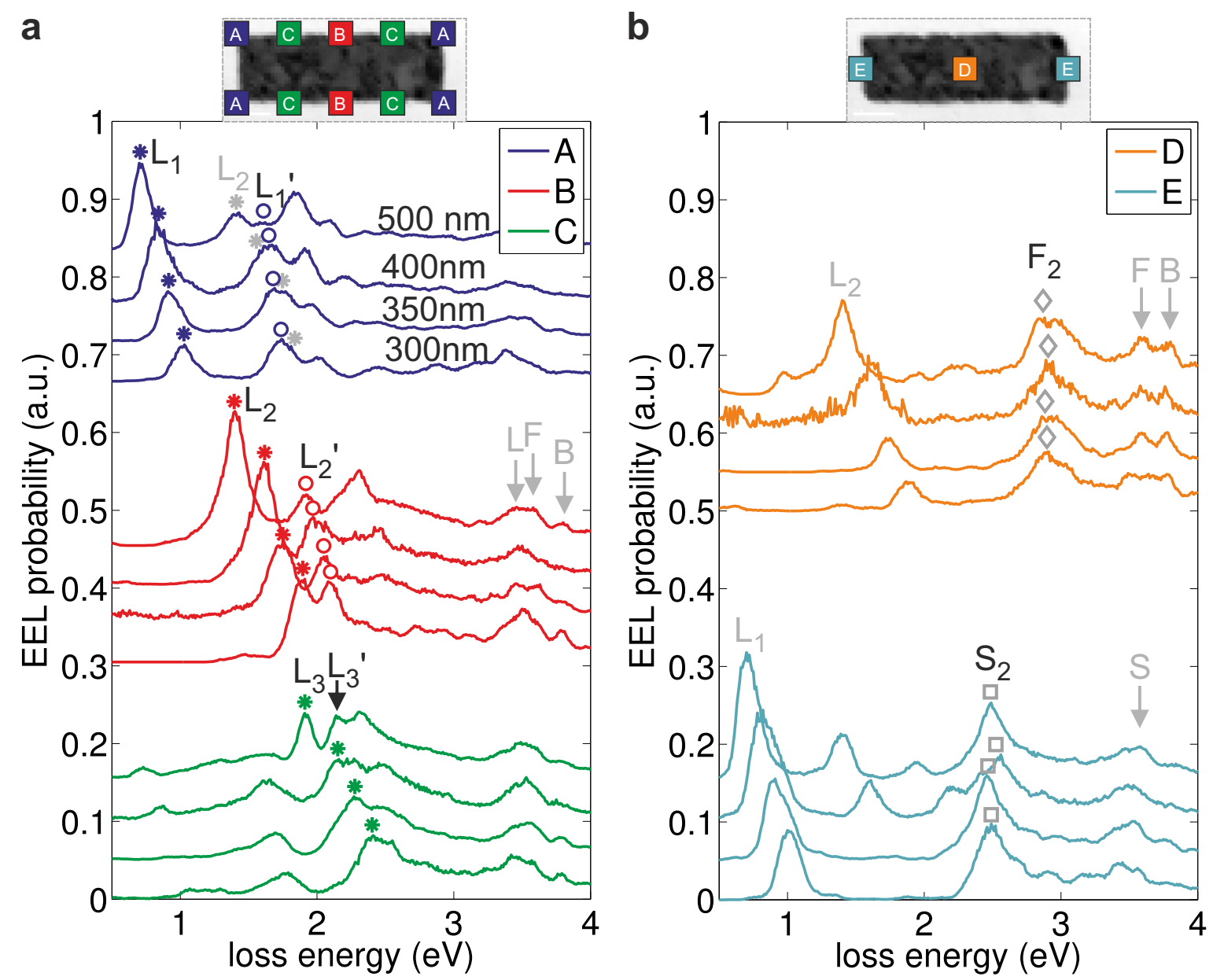

Supplementary Figure 1: Deconvolved EEL spectra of edge and film modes for cuboids with lengths of 300, 350, 400 and $500 \mathrm{~nm}$ (compare Figure 1 in the main text). (a) Spectra taken along the long cuboid edge, the respective areas are indicated by the letters in the inset electron micrograph (data from like letters are averaged). $\mathrm{L}_{x}$ and $\mathrm{L}_{x}^{\prime}(\mathrm{x}=1,2,3)$ are the edge modes along the long cuboid axis of first to third order, $\mathrm{L}$ is the corresponding asymptotic edge mode. (b) Spectra taken along the short edge and from the cuboid center. $\mathrm{S}_{2}$ is the second order edge mode along the short cuboid axis, $\mathrm{F}_{2}$ is the second order film mode along the short cuboid axis and $\mathrm{S}$ is the corresponding asymptotic edge mode. In both panels, the EEL peaks are indicated by the same symbols as used in Figure 4 in the main text. $F$ is the asymptotic film mode and B is the bulk plasmon mode. 


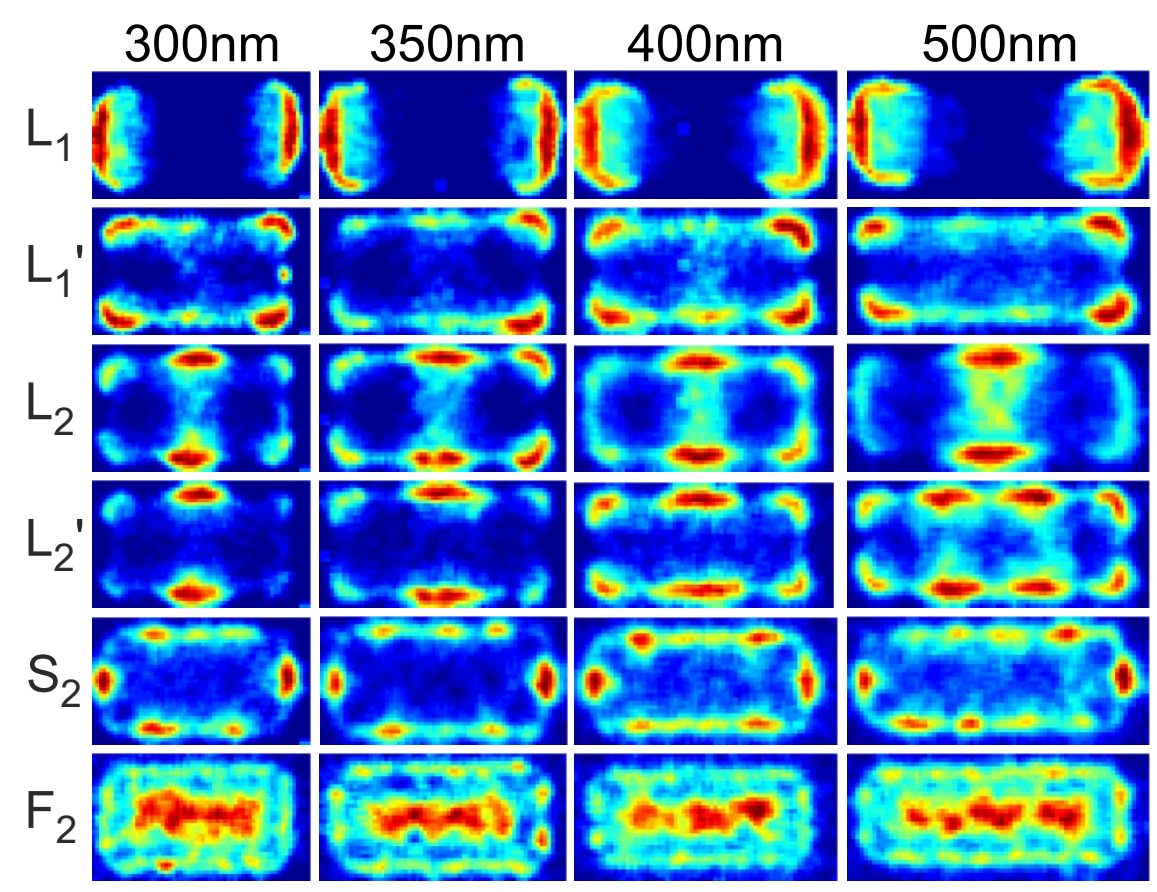

Supplementary Figure 2: Measured EEL maps for cuboids with lengths of 300, 350, 400 and $500 \mathrm{~nm}$. EEL maps for edge modes $\mathrm{L}_{1}, \mathrm{~L}_{1}^{\prime}, \mathrm{L}_{2}$ and $\mathrm{L}_{2}^{\prime}$ along the long cuboid axes and edge and film modes $\mathrm{S}_{2}$ and $\mathrm{F}_{2}$ along the short cuboid axis. The center loss energies of the used $100 \mathrm{meV}$ energy window correspond to the peak energies in Figure 4 in the main text and in Supplementary Figure 1, with the exception of the $L_{1}^{\prime}$ maps for the cuboid lengths 350,400 and $500 \mathrm{~nm}$. In these cases, the maps are generated at $0.1 \mathrm{eV}$ lower $(350 \mathrm{~nm})$, $0.15 \mathrm{eV}$ higher $(400 \mathrm{~nm})$ and $0.2 \mathrm{eV}$ higher energies $(500 \mathrm{~nm})$ than the corresponding peak energies (compare Supplementary Figure 1). This is done to separate the spectrally close mode patterns of the $\mathrm{L}_{1}^{\prime}$ and $\mathrm{L}_{2}$ modes. We note that this was not possible for the $\mathrm{L}_{2}^{\prime}$ map for the cuboid with a length of $500 \mathrm{~nm}$, due to the identical peak energy of the $\mathrm{L}_{3}$ mode that dominates the observed pattern. However, the locally extracted spectrum (top red curve in Supplemetary Figure 1) strongly hints towards the existence of the specific $\mathrm{L}_{2}^{\prime}$ mode. The distinct EEL map patterns for each mode, independent of the cuboid length, corroborate our mode assignments. 


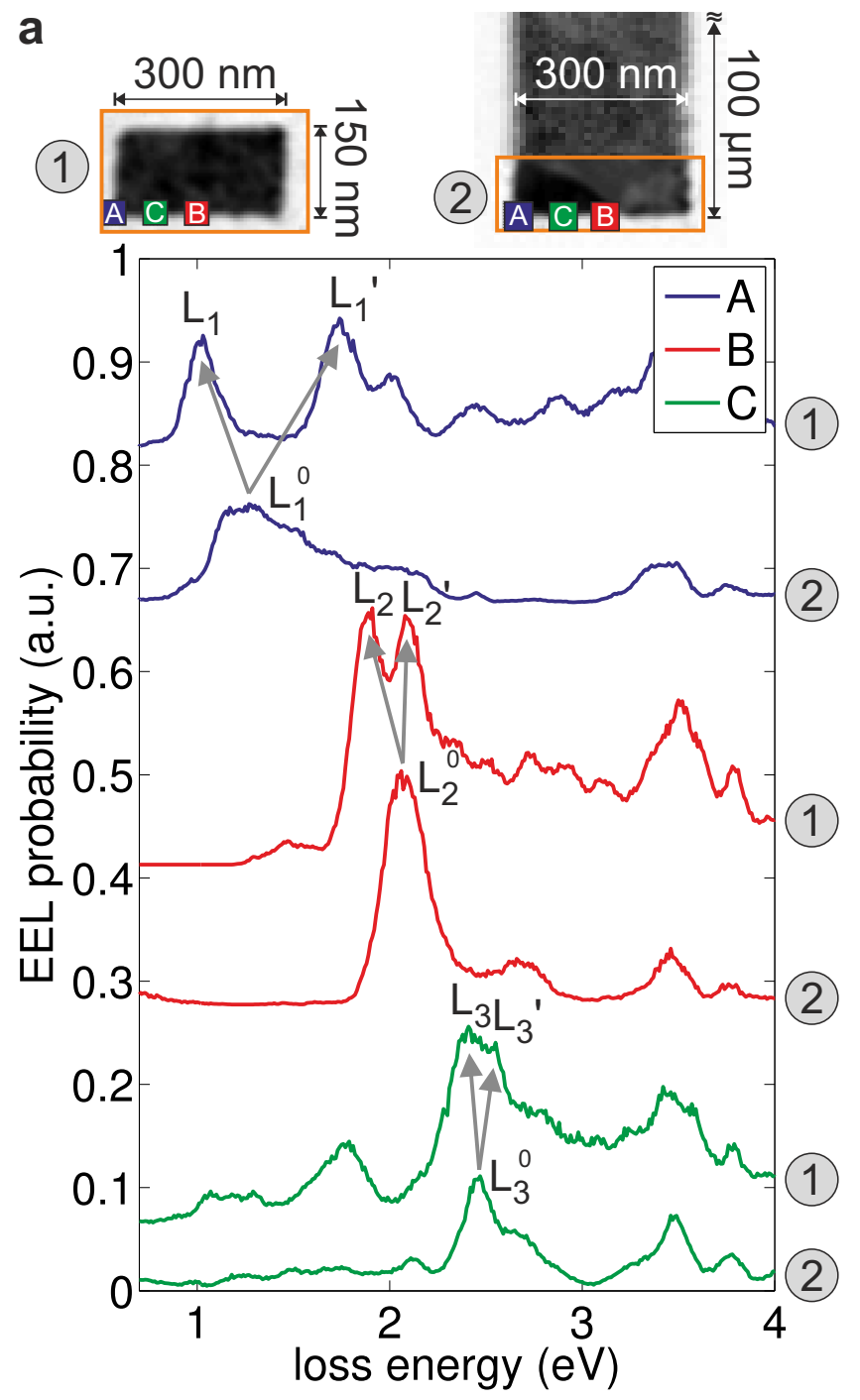

b
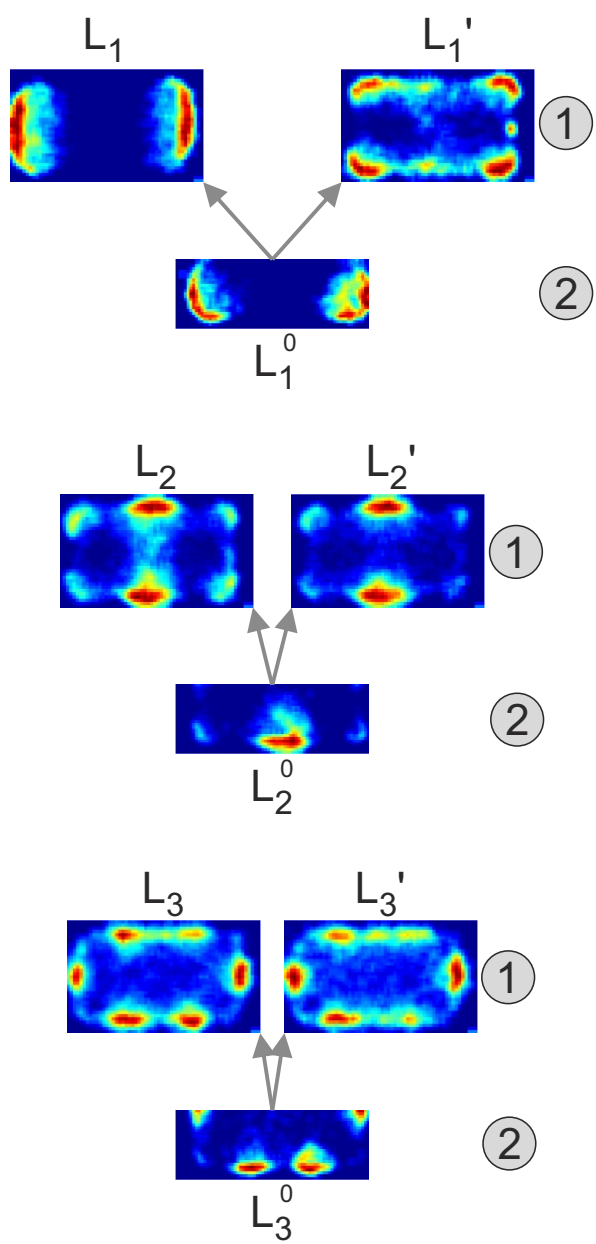

Supplementary Figure 3: Comparison of coupled and uncoupled plasmon modes along a $300 \mathrm{~nm}$ edge. (a) Deconvolved EEL spectra taken along the long cuboid edge with a length of $300 \mathrm{~nm}$, the respective areas are indicated by the letters in the inset electron micrograph (1), highlighting the mode splitting of the edge modes $\mathrm{L}_{1}^{0}-\mathrm{L}_{3}^{0}$ into $\mathrm{L}_{1}-\mathrm{L}_{3}$ and $\mathrm{L}_{1}^{\prime}-\mathrm{L}_{3}^{\prime}$ (gray arrows) due to plasmon coupling of the opposing edges. These spectra are compared to deconvolved EEL spectra taken along an edge of similar length but much larger width $(100 \mu \mathrm{m}$ instead of $150 \mathrm{~nm}$ ), thus hindering plasmon coupling and therefore mode splitting (2). (b) EEL maps extracted at loss energies corresponding to modes $\mathrm{L}_{1}-\mathrm{L}_{3}$ and $\mathrm{L}_{1}^{\prime}-\mathrm{L}_{3}^{\prime}(1)$ and $\mathrm{L}_{1}^{0}-\mathrm{L}_{3}^{0}(2)$ respectively, as given by the highlighted peaks in (a). The energy window of each EEL map is $100 \mathrm{meV}$. 